DIGITALCOMMONS — @WAYNESTATE-
Clinical Research in Practice: The Journal of Team Hippocrates

2019

\title{
Renal angioplasty does not improve clinical outcomes compared to standard medical therapy in patients with atherosclerotic renal artery stenosis
}

Alexandra Morris

Wayne State University School of Medicine, almorris@med.wayne.edu

Follow this and additional works at: https://digitalcommons.wayne.edu/crp

Part of the Internal Medicine Commons, Medical Education Commons, and the Translational Medical Research Commons

\section{Recommended Citation}

MORRIS A. Renal angioplasty does not improve clinical outcomes compared to standard medical therapy in patients with atherosclerotic renal artery stenosis. Clin. Res. Prac. 2019 Feb 6;5(1):eP1689. doi: 10.22237/crp/1549411380

This Critical Analysis is brought to you for free and open access by the Open Access Journals at DigitalCommons@WayneState. It has been accepted for inclusion in Clinical Research in Practice: The Journal of Team Hippocrates by an authorized editor of DigitalCommons@WayneState. 


\title{
Renal angioplasty does not improve clinical outcomes compared to standard medical therapy in patients with atherosclerotic renal artery stenosis
}

ALEXANDRA MORRIS, Wayne State University School of Medicine, almorris@med.wayne.edu

\begin{abstract}
A critical appraisal and clinical application of Cooper CJ, Murphy T, Cutlip D, Jamerson K, Henrich W. Stenting and medical therapy for atherosclerotic renal-artery stenosis. New Eng J Med. 2014;370(1):13-22. doi: 10.1056/NEJMoa1310753.
\end{abstract}

Keywords:

renal artery stenosis, hypertension

\section{Clinical Context}

TR is a 56-year-old African American male with a past medical history significant for hypertension who presented to the emergency department with a 1-day history of headache and chest pain. Upon presentation he was diagnosed with hypertensive emergency with blood pressures of 200s/100s, an elevated creatinine, and signs of myocardial ischemia with elevated troponin. The patient's blood pressure was refractory to multiple antihypertensive agents. A computed tomography angiography was performed, which demonstrated evidence of $>80 \%$ ostial stenosis of the renal arteries bilaterally. At this time renal artery angioplasty and stenting was recommended to the patient. The patient was imprisoned 2 months prior and during incarceration he was nonadherent with his 3 blood pressure medications. The patient was hesitant about the procedure and asked if he could continue his current blood pressure medication regimen with improved adherence instead of undergoing the renal angioplasty procedure.

\section{Clinical Question}

Is renal angioplasty superior to standard medical treatment in reducing hypertension and improving cardiac and renal clinical outcomes in patients with renal artery stenosis?

\section{Research Article}

Cooper CJ, Murphy T, Cutlip D, Jamerson K, Henrich W. Stenting and medical therapy for atherosclerotic renal-artery stenosis. New Eng J Med. 2014;370(1):13-22. doi: 10.1056/NEJMoa1310753

ALEXANDRA MORRIS is a medical student at Wayne State University School of Medicine. 
MORRIS A. Renal angioplasty does not improve clinical outcomes compared to standard medical therapy in patients with atherosclerotic renal artery stenosis. Clin. Res. Prac. 2019 Feb 6;5(1):eP1689. doi: $10.22237 / \mathrm{crp} / 1549411380$
Clinical Research in Practice The Journal of Team Hippocrates

VOL 5 ISS 1 / eP1689 / FEBRUARY 6, 2019 doi: $\underline{10.22237 / \mathrm{crp} / 1549411380}$

\section{Related Literature}

A literature search was performed with the keywords "renal artery stenosis treatment" in both PubMed and Google scholar. In order to further narrow down the search, all case reports were excluded, and meta-analyses were selectively reviewed based on their date of publication, and their citations used to guide further searches. $1,2,3$ Other keywords used to refine the search of pertinent studies included "renal revascularization" and "renal angioplasty" with and without stenting, specifically comparing the effectiveness of this treatment option with medical therapy. This search led to a comprehensive report published in 2013 by the American College of Cardiology Foundation/American Heart Association. ${ }^{4}$ This report provided guidelines pertaining to revascularization in patients with renal artery stenosis (RAS) and its associated level of evidence, which was used to further guide the search to look for the most impactful studies that this evidence was based on.

In the 1990s multiple uncontrolled retrospective studies suggested significant reductions in systolic blood pressure after renal artery angioplasty ${ }^{5}$, which increased the rate of this procedure. However, during this time 3 randomized trials demonstrated that renal artery angioplasty actually failed to show a benefit over standard medical therapy. $., \underline{z}, \underline{9}$

In 1998 two papers were published that compared percutaneous angioplasty with medical therapy for hypertensive patients with renal artery stenosis due to atherosclerosis, comparing both blood pressure control and incidence of complications. $-\mathbf{6}, \mathbf{B}$ Both studies demonstrated no increased improvement in blood pressure with renal angioplasty over medical therapy and showed that intervention had significant complication rates. One of the studies performed by Webster et al. was flawed in that it included a broad definition of RAS as stenosis $>50 \%$, however only a stenosis $>75 \%$ or $>60 \%$ with post-stenotic dilatation suggests that the lesion may be producing hemodynamically significant reductions in renal blood flow causing hypertension (HTN). - Furthermore, the study is outdated and may overestimate the incidence of serious complications attributable to the procedure (bleeding at the entry site, renal infarction, restenosis, and death) in light of what occurs today with improved technology and expertise.

The other study in 1998 performed by Plouin et al. was not used for analysis because it excluded patients with myocardial infarction, which our patient suffered. Additionally, only 49 patients were enrolled leading to a small effect size. Furthermore, this study focused on unilateral renal artery stenosis, which doesn't apply to our patient with bilateral RAS. Lastly, the medical therapy that was used to compare against renal angioplasty was not similar to the typical regimen today. For instance, blockade of the reninangiotensin-aldosterone pathway is known to be highly effective in controlling blood pressure as well as improving renal and cardiovascular outcomes $\stackrel{8}{ }$, however the use of these drugs was restricted in this study due to the fear of acute renal failure.

In 2000 Van Jaarsveld et al. published Dutch Renal Artery Stenosis Intervention Cooperative (DRASTIC) study, a randomized trial comparing percutaneous transluminal renal angioplasty and drug therapy. ${ }^{9}$ The authors concluded that angioplasty has little advantage over antihypertensive-drug therapy in patients with renal artery stenosis. This study was flawed in that almost $40 \%$ of the study's patients originally enrolled in the medical treatment group switched to the angioplasty group within the first 3 months. However, patients were still analyzed as part of the group to which they were initially randomized. This study was not chosen for analysis since more recent and robust trials have since been performed.

In 2009, the ASTRAL trial was a large randomized controlled trial comparing the clinical benefit of revascularization versus medical therapy. ${ }^{10}$ This trial was not selected for analysis because patients were only enrolled in the trial if the physician was uncertain as to whether revascularization would provide a worthwhile clinical benefit, therefore the majority of these patients did not have true indications for renal artery angioplasty, unlike our patient. In the same year the STAR trial was published showing that renal artery stenting did not improve renal function more than medical management and resulted in significant procedure-related complications. $\stackrel{14}{ }$ This trial was not used for analysis because many patients were falsely identified as having renal artery stenosis $>50 \%$ by noninvasive imaging and the end-points of this study were limited compared to other trials.

Interestingly, 3 studies showed superiority of revascularization procedures over antihypertensive medications in instances of renal artery stenosis with flash pulmonary edema ${ }^{11}$, chronic kidney disease (CKD) $\underline{\underline{12}}$, and refractory heart failure..$^{\underline{13}}$ Our patient did not experience any of these complications and therefore these studies were not chosen for analysis.

The current American College of Cardiology/American Heart Association guidelines published in 2013 mentioned above were mostly based on preliminary data from the Cardiovascular Outcomes in Renal Atherosclerotic Lesions (CORAL) trial published in 2014 by 
MORRIS A. Renal angioplasty does not improve clinical outcomes compared to standard medical therapy in patients with atherosclerotic renal artery stenosis. Clin. Res. Prac. 2019 Feb 6;5(1):eP1689. doi: $10.22237 / \mathrm{crp} / 1549411380$
Clinical Research in Practice The Journal of Team Hippocrates

VOL 5 ISS 1 / eP1689 / FEBRUARY 6, 2019 doi: $\underline{10.22237 / \mathrm{crp} / 1549411380}$

Cooper et al. $\underline{15}$ This study was selected for this appraisal because it is a relatively recent, large, multicenter randomized trial that studied patients with atherosclerotic renal artery stenosis receiving either renal artery stenting and medical therapy or medical therapy alone; showing that renal artery angioplasty did not confer a significant benefit in preventing adverse cardiovascular or renal events. This study was also novel in that the investigators used strict guidelines for medical therapy that included good control of blood pressure (BP), treatment of dyslipidemia and diabetes, smoking cessation, administration of an antiplatelet agent, and treatment of complications of renal insufficiency that was based on current, evidence-based practice guidelines. Furthermore, this trial included patients with bilateral renal artery stenosis, similar to our patient. Additionally, a paper was published examining the rationale and design of the CORAL trial, which demonstrated that the study would have a $90 \%$ power to detect a $28 \%$ reduction in primary end point hazard rate, which was significant to determine if stent revascularization was superior to optimal medical therapy. $\frac{16}{}$ A Cochrane review of eight randomized controlled trials also supported the conclusions drawn from the CORAL trial, which will be critically appraised below. $\underline{17}$

\section{Critical Appraisal}

Cooper et al. conducted a multicenter, open-label, randomized, controlled trial comparing medical therapy alone vs. medical therapy with renal artery stenting on adverse cardiovascular and renal events in patients with atherosclerotic renal artery stenosis. This article is considered a strength of recommendation taxonomy (SORT) level 1 study because it is a randomized control trial with data from multiple different clinical centers. The CORAL trial was primarily funded by the National Heart, Lung, and Blood Institute. However, equipment and medication donation as well as supplemental financial support was provided by AstraZeneca, Pfizer, and Cordis, although evidence of funding bias is minimal.

The study included 947 patients with severe renal artery stenosis with hypertension, measured as a systolic BP of $155 \mathrm{~mm} \mathrm{Hg}$ or higher on at least two anti-hypertensive medications. Severe renal artery stenosis was defined as a stenosis of at least $80 \%$ or stenosis of at least $60 \%$ with a systolic pressure gradient of at least $20 \mathrm{~mm} \mathrm{Hg}$. Our patient had bilateral stenosis of greater than $80 \%$, which is considered severe. Angiograms measuring the stenosis were validated with a computerized quantitative vascular analysis program. There were changes to the enrollment requirements during the course of the trial from 2005 to 2010 , potentially affecting the results. Later on in the study, the threshold of $155 \mathrm{~mm} \mathrm{Hg}$ for defining systolic HTN was no longer specified due to slower than expected patient enrollment. Also, patients with RAS without HTN could be enrolled if they had CKD, which is not pertinent to our patient's specific clinical question. Patients were excluded from the study if they had RAS due to fibromuscular dysplasia, CKD from a cause other than ischemic nephropathy or associated with creatinine higher than 4, kidney length of less than $7 \mathrm{~cm}$, and a lesion that could not be treated with the use of a single stent. 16 patients were excluded from the results owing to scientific integrity concerns and 143 patients were lost to follow-up; however despite the discontinuation rates the number of patients used in analysis was still enough to maintain significance. Our patient fit into the inclusion criteria used in this study.

Patients were evenly randomly assigned to stenting in addition to medical therapy or medical therapy alone with the primary end points being the occurrence of a major cardiovascular or renal event which were a composite of death from cardiovascular or renal causes, stroke, MI, hospitalization for $\mathrm{CHF}$, progressive renal insufficiency, or dialysis requirement. Randomization was performed using an interactive voice randomization system with the use of a permuted block design, which was successful since there were no significant demographic differences between the two groups at baseline. There was no blinding involved due to the fact that one of the treatments was a procedure and the other was medication based, which could have led to some bias in reporting of the end points. All participants in the study received antiplatelet therapy and other protocol-derived medical therapies to control BP, glucose, and lipid levels. Participants were followed for a median of 43 months. With 947 patients and follow-up through 2012 , the study has a $90 \%$ power to test the hypothesis that stenting would reduce the incidence of the primary end point by $25 \%$ (Hazard ratio, 0.75 ) at 2 years, at a two-sided type I error rate of 0.05 .

In patients receiving renal angioplasty, there was a significant reduction of the stenosis from $68+/-11 \%$ to $16+/-8 \%$ ( $p<0.001$ ). The most common complication that occurred from stenting was arterial dissection, which occurred in 11 patients. Systolic BP declined in both the medical-therapy-only group and stent group, but modestly lower in the stent group by about $2 \mathrm{~mm} \mathrm{Hg}(\mathrm{p}=0.03$ ). However, there was no significant difference in the occurrence of the primary composite end point between the stent group and medical-therapy-only group (35.1\% and $35.8 \%$ respectively, $\mathrm{HR}=0.94,95 \% \mathrm{Cl}$ : 0.76 to $1.17, \mathrm{p}=0.58)$. 
MORRIS A. Renal angioplasty does not improve clinical outcomes compared to standard medical therapy in patients with atherosclerotic renal artery stenosis. Clin. Res. Prac. 2019 Feb 6;5(1):eP1689. doi: $10.22237 / \mathrm{crp} / 1549411380$
Clinical Research in Practice The Journal of Team Hippocrates

VOL 5 ISS 1 / eP1689 / FEBRUARY 6, 2019 doi: $10.22237 / \mathrm{crp} / 1549411380$

This study has limitations that affect the ability to replicate this treatment protocol in clinical practice. The medical therapy in the study included the use of angiotensin II receptor blocker (ARB, specifically candesartan) with or without a thiazide type diu retic, amlodipine, anti-platelet therapy, and atorvastatin; therefore these patients had good cardiovascular and renal outcomes despite their advanced age and high rates of HTN, diabetes mellitus (DM), CKD, and other co-existing cardiovascular conditions. The medical therapy used in this trial follows standard clinical practice guidelines, however it is important to note that the specific drugs used, such as candesartan, are sold by the companies funding the research. Moreover, this medication regimen may not be relevant to the population we serve since many of our patients, such as the one described above, do not have access to primary care and do not have well controlled chronic medical conditions. Furthermore, only $7 \%$ of the study population was African American like our patient, and the average age of participants was 69 years old, which is significantly older than our patient. There is evidence of selection bias in the CORAL study since many patients were likely not enrolled in the study if physicians thought they needed stent placement because the average renal artery stenosis was moderate at $67 \%$. In addition, there is still some debate on what percentage of stenosis warrants intervention. Thus the major limitation was the inability to select hemodynamically severe renal artery stenosis for treatment. However, the authors argue that the study did not show differences between groups among participants with $>80 \%$ stenosis, which is the most relevant to this patient case.

\section{Clinical Application}

The study discussed in the critical appraisal is an appropriate and useful study that is able to inform the clinical decision-making of this patient. The CORAL study was a reliable study with a large sample size that found that there was no clinical benefit of stenting over medical management with respect to the rate of cardiovascular and renal clinical outcomes. There was a modest decrease in systolic BP with stenting that was statistically significant but not clinically significant. The investigators of this study reasonably concluded that medical management should be the primary treatment of atherosclerotic renal artery stenosis. The inclusion criteria of this study closely matched our patient, however all of the patients in the study were outpatient and our patient acutely presented to the hospital in hypertensive emergency, therefore the results cannot be entirely generalized. In our case, the patient wanted to avoid the renal angioplasty procedure due to the associated potential risks of infection, thrombosis, and renal artery dissection. Instead he desired medical therapy, which is consistent with the current recommendations.

Learning points:

1. For patients with bilateral atheromatous renal artery stenosis, medical therapy is successful in improving clinical outcomes and should be considered the first-line treatment.

2. All patients with hypertension from renal artery stenosis should be medically optimized with anti-platelet therapy and control of blood pressure, glucose, and lipid levels to prevent adverse cardiovascular and renal events.

3. Since renal artery angioplasty offers no clinical advantage over medical management and has many associated risks, patients should not be offered this based on a history of non-adherence and instead shared decisionmaking should be made and medical optimization must be performed.

\section{References}

1. Balk E, Raman G, Chung M, et al. Effectiveness of management strategies for renal artery stenosis: a systematic review. Ann Intern Med. 2006 Dec 19;145(12):901-12. doi: 10.7326/0003-4819-145-12-200612190-00143

2. Isles CG, Robertson S, Hill D. Management of renovascular disease: a review of renal artery stenting in ten studies. QJM: An International Journal of Medicine. 1999;92(3):159-167. doi: 10.1093/qjmed/92.3.159

3. Perkovic V. Review: revascularization and medical treatment may be similar in atherosclerotic renal artery stenosis. Ann Intern Med. 2016;165(12). doi: 10.7326/ACPJC-2016-165-12-071 
MORRIS A. Renal angioplasty does not improve clinical outcomes compared to standard medical therapy in patients with atherosclerotic renal artery stenosis. Clin. Res. Prac. 2019 Feb 6;5(1):eP1689. doi: $10.22237 / \mathrm{crp} / 1549411380$
Clinical Research in Practice The Journal of Team Hippocrates

VOL 5 ISS 1 / eP1689 / FEBRUARY 6, 2019 doi: $\underline{10.22237 / \mathrm{crp} / 1549411380}$

4. Anderson JL, Halperin JL, Albert NM, et al. Management of patients with peripheral artery disease (compilation of 2005 and 2011 ACCF/AHA Guideline Recommendations): A report of the American College of Cardiology Foundation/American Heart Association task force on practice guidelines. Circulation. 2013 Apr 2;127(13):1425-43. doi: 10.1161/CIR.0b013e31828b82aa

5. lannone LA, Underwood PL, Nath A, Tannenbaum MA, Ghali MGH, Clevenger LD. Effect of primary balloon expandable renal artery stents on long-term patency, renal function, and blood pressure in hypertensive and renal insufficient patients with renal artery stenosis. Catheter Cardiovasc Interv. 1996;37(3):243-250. doi: 10.1002/(SICI)1097-0304(199603)37:3<243::AIDCCD3>3.0.CO;2-C

6. Webster J, Marshall F, Abdalla M, et al. Randomised comparison of percutaneous angioplasty vs continued medical therapy for hypertensive patients with atheromatous renal artery stenosis. Scottish and Newcastle Renal Artery Stenosis Collaborative Group. J Hum Hypertens. 1998 May;12(5):329-35. doi: 10.1038/sj.jhh.1000599

7. Plouin PF, Chatellier G, Darne B, Raynaud A. Blood pressure outcome of angioplasty in atherosclerotic renal artery stenosis: a randomized trial. Essai Multicentrique Medicaments vs Angioplastie (EMMA) Study Group. Hypertension. 1998 Mar;31(3):823-9. doi: 10.1161/01.hyp.31.3.823

8. Dworkin LD, Cooper CJ. Renal-Artery Stenosis. New Eng J Med. 2009;361(20):1972-1978. doi: 10.1056/nejmcp0809200

9. Van Jaarsveld BC, Krijnen P, Pieterman H, Derkx F, Deinum J. The effect of balloon angioplasty on hypertension in atherosclerotic renal-artery stenosis. Dutch Renal Artery Stenosis Intervention Cooperative Study Group. New Eng J Med. 2000;342(14):10071014. doi: $10.1056 /$ nejm200004063421403

10. Wheatley K, Ives N, Gray R, et al. Revascularization versus medical therapy for renal-artery stenosis. New Eng J Med. 2009; 361(20):1953-1962. doi: 10.1056/nejmoa0905368

11. Ritchie J, Green D, Chrysochou C, Chalmers N, Foley RN, Kalra PA. High-risk clinical presentations in the atherosclerotic renovascular disease: prognosis and response to renal artery revascularization. Am J Kidney Dis. 2014 Feb;63(2):186-97. doi: 10.1053/i.ajkd.2013.07.020

12. Kalra PA, Chrysochou C, Green D, Cheug CM, Khavandi K, Sixt S, Rastan A, Zeller T. The benefit of renal artery stenting in patients with atheromatous renovascular disease and advanced chronic kidney disease. Catheter Cardiovasc Interv. 2010 Jan 1;75(1):110. doi: $10.1002 /$ ccd. 22290

13. Gray BH, Olin JW, Childs MB, Sullivan TM, Bacharach JM. Clinical benefit of renal artery angioplasty with stenting for the control of recurrent and refractory congestive heart failure. Vasc Med. 2002;7(4):275-9. doi: 10.1191/1358863x02vm456oa

14. Bax L, Arend-Jan J, Kouwenberg HJ, et.al. Stent placement in patients with atherosclerotic renal artery stenosis and impaired renal function: a randomized trial. Ann Intern Med. 2009 Jun 16;150(12):840-8, W150-1. doi: 10.7326/0003-4819-150-12200906160-00119

15. Cooper CJ, Murphy T, Cutlip D, Jamerson K, Henrich W. Stenting and medical therapy for atherosclerotic renal-artery stenosis. New Eng J Med. 2014;370(1):13-22. doi: 10.1056/NEJMoa1310753

16. Cooper CJ, Murphy TP, Matsumoto A, et al. Stent revascularization for the prevention of cardiovascular and renal events among patients with renal artery stenosis and systolic hypertension: rationale and design of the CORAL trial. Am Heart J. 2006 Jul;152(1):59-66. doi: 10.1016/j.ahj.2005.09.011

17. Jenks S, Yeoh SE, Conway BR. Balloon angioplasty, with and without stenting, versus medical therapy for hypertensive patients with renal artery stenosis. Cochrane Database Syst Rev (2014) 12:CD002944. doi: 10.1002/14651858.CD002944.pub2 